\title{
Flaws Characterization with Pulsed Eddy Currents N.D.T.
}

\author{
T.Clauzon, F.Thollon, A. Nicolas. \\ C.E.G.E.L.Y-UPRESA C.N.R.S 5005-Ecole Centrale de Lyon. B.P163-69131 Ecully cedex-France.
}

\begin{abstract}
In this paper, we present a method for characterizing flaws in metallic structures with pulsed eddy current non-destructive testing (NDT). In a first step, we explain why we have chosen a deconvolution method based on Conjugate Gradient algorithm (CG). After a description of this method and the way it is used for characterization, a validation is performed by coupling it with a 2D FEM ( 2 dimensional finite elements method) package. The obtained results are compared with experimental measurements. In the last step, a parametric study on parameters obtained with the impulse response gives an estimation of the dimensions of the flaw.
\end{abstract}

Index terms - Eddy current testing, flaw characterization, deconvolution.

\section{INTRODUCTION}

The development of N.D.T in aeronautical structures has a considerable importance today. We present results on the characterization of deep flaws in riveted assemblies (Fig.1). These assemblies are composed of aluminum sheets linked with titanium rivets, with a filler between each sheet. The flaws appear under the filler and near the rivet running parallel to it. Due to the filler, ultrasounds do not give results. So pulsed eddy current N.D.T has to be used. Characterization is quite difficult due to the number of parameters, the bad knowledge of physical properties of the material under control, and the low level of measurement. A way for the characterization consists in searching impulse response of the system (sensor +spare +flaw) in order to have information on magnitude and phase versus frequency. We have chosen the impulse response because it does not depend on the shape of the excitation signal. In this purpose, we use a deconvolution method by Conjugate Gradient, which gives directly the impulse response of the system, combined with a 2D FEM package.

\section{PRINCIPLE OF THE METHOD}

The impulse response $h(t)$ for a linear causal system is linked to the input $x(t)$ and output $y(t)$ by the convolution operation:

$$
y(t)=\int_{0}^{\infty} x(t-\tau) h(\tau) d \tau .
$$

Deconvolution implies the inversion of equation (1). By discretizing (1), we obtain:

Manuscript received June 1, 1998.

$$
y(m)=K \sum_{k=0}^{n-1} x(m-k) h(k),
$$

with $m=t / T_{c}$, and where $K$ is a normalization constant, $m$ the number of samples and $T e$ the sample period. $K$ is chosen equal to the sample period $T e$. This equation can be written under a linear system form:

$$
\mathbf{Y}=\mathbf{A H} \text {. }
$$

$\mathbf{Y}$ and $\mathbf{H}$ are vectors containing respectively output signal $y(t)$ and impulse response $h(t)$. A is a matrix [3] build with the input signal $x(t)$.

The basic philosophy of the CG is to minimize the functional $F(h)$ given by [1]:

$$
F(h)=\|\mathbf{A H}-\mathbf{Y}\|^{2} \text {, }
$$

instead solving $\mathbf{H}=\mathbf{A}^{-1} \mathbf{Y}$ directly.

In our case, the $\mathrm{CG}$ algorithm is not applied for the resolution of system (3), but directly to the convolution operator defined by:

$$
C=\int_{0}^{\infty} x(t-\tau)(*) d \tau,
$$

because it allows working only with vectors. Furthermore, as it is not necessary to build matrix $\mathbf{A}$, the method brings about a certain saving in memory and computational time.

The CG method starts with an initial guess value $\mathbf{H}_{\mathbf{o}}$ (generally chosen equal to $O$ ) and generates [1]-[2]:

$$
\begin{gathered}
\mathbf{R}_{0}=\left(C \mathbf{H}_{0}-\mathbf{Y}\right) \\
\mathbf{P}_{0}=-b_{0} C^{*} \mathbf{R}_{0} ; b_{0}=1 /\left\|C^{*} \mathbf{R}_{0}\right\|^{2}
\end{gathered}
$$

where $C^{*}$ represents the adjoint operator [1], which is defined as:

$$
C^{*} z(t)=\int_{0}^{\infty} x(t-\tau) z(t) d t .
$$

Then the CG develops according to the following:

$$
\begin{aligned}
& \mathbf{H}_{k+1}=\mathbf{H}_{k}+\alpha_{k} \mathbf{P}_{k} \\
& \mathbf{R}_{k+1}=\mathbf{R}_{k}+\alpha_{k} C \mathbf{P}_{k}, \\
& \mathbf{P}_{k+1}=\mathbf{P}_{k}-b_{k+1} C^{*} \mathbf{R}_{k+1}
\end{aligned}
$$

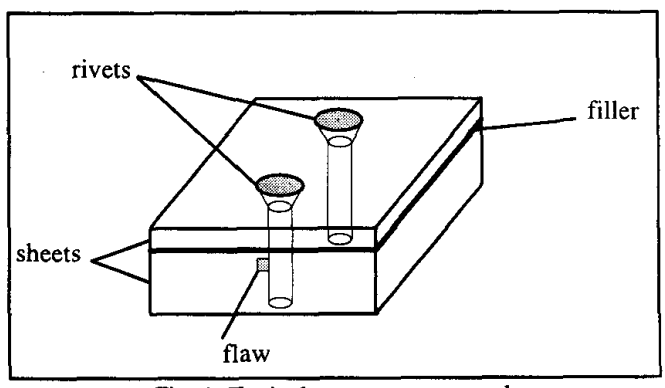

Fig. 1. Typical structure to control. 
with $\alpha_{k}=\frac{1}{\left\|C \mathbf{P}_{k}\right\|^{2}} ; b_{k}=\frac{1}{\left\|C \mathbf{R}_{k}\right\|^{2}}$.

The CG algorithm can be speed up using F.F.T for the computation of the convolution.

The process must be stopped before error on the data $x(t)$ and $y(t)$ (i.e. measurement noise) deteriorates the resolution of the impulse response $h$. In this purpose, we define a stopping criterion base on the magnitude of maximum error value on $x(t)$ and $y(t)$. We put $\Delta x$ and $\Delta y$ respective error on $x$ and $y$; residue $R$ can be written as:

$$
R(t)=\int_{0}^{\infty} x(t-\tau) h(\tau) d \tau-y(t)+\int_{0}^{\infty} \Delta x h(\tau) d \tau-\Delta y,
$$

hence:

$$
R(t)=\int_{0}^{\infty} \Delta x h(\tau) d \tau-\Delta y,
$$

which implies:

$$
|R(t)| \leq \int_{0}^{\infty} \Delta x h(\tau) d \tau|+| \Delta y \mid
$$

Assuming that:

$$
|\Delta x| \leq B ; \quad|\Delta y| \leq B,
$$

(where $B$ is a constant defined as the largest value of the noise on $x$ and $y$ ), we obtain:

$$
|R(t)| \leq B\left\{\int_{0}^{T}|h(t)| d t+1\right\} .
$$

Note that we have replaced the upper limit in (14) by $T$ (length of the signal $h(t)$ ), because in practice the data is recorded up to a finite time $T$.

It was shown in [1] and [2] that the above inequality is a necessary and sufficient condition for $h(t)$ to be a solution of (3). The CG algorithm is stopped when we found a residue $R$ that satisfies inequality (14). In order to remove the noise on the measured data, we have slightly modified the initial CG algorithm by weighting the residue [2] with an exponential function $f=\exp (-t / 0.02)$.

\section{STUDIED STRUCTURE}

The studied structure is an aluminum cylinder where a flaw is machined. The sensor is composed with an optimized coil [3] and measurement quantity is horizontal flux density. The excitation signal is a square shape current with variable duty cycle. Simulations are realized with the Fissure package, which used a two dimensional finite elements method. The structure in Fissure is described with an axially symmetric geometry (Fig.2). For the experimental system (Fig.3), a set

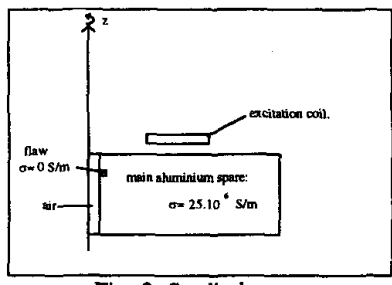

Fig. 2. Studied geometry.

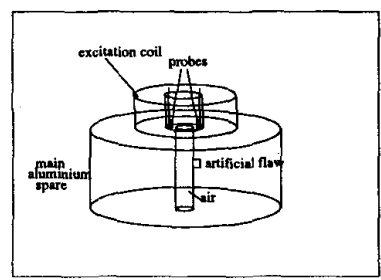

Fig. 3. Experimental system of test samples was realized. To be representative of the riveted structures, each sample is composed of an aluminum cylinder with a central hole. The sensor uses separated elements for induction and detection. A coil creates an electromagnetic field to induce eddy current into the sample under test. For the detection, two Hall probes are used, one upon a region with a flaw and another upon a region supposed to be without a flaw. Then, the measurement quantity is the difference between the two Hall effect signals. The measurement point is located at $5 \mathrm{~mm}$ from the symmetric axis.

\section{RESULTS}

We study first the influence of the lift-off. The curves (Fig.4) represent the impulse responses normalized in magnitude ( $\vec{u} /\|\vec{u}\|)$ for two different lift-off. As we have the same results, we can conclude that a variation of the lift-off modifies only the magnitude of the response. Then we study 3 kinds of flaws (respectively (length $\times$ heigth):

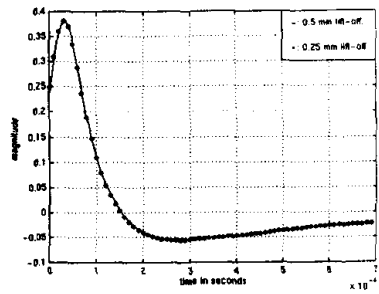

Fig. 4. Impulse response
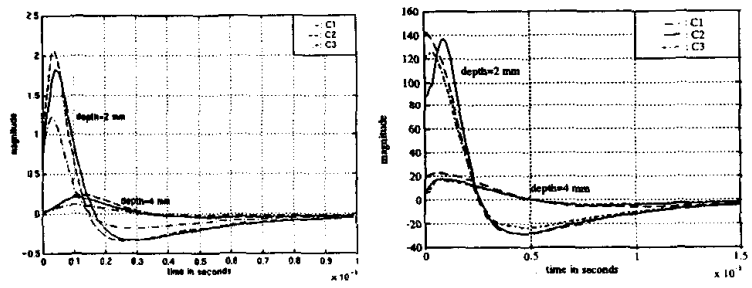

Fig. 5. Impulse response respectively simulation and measurement.
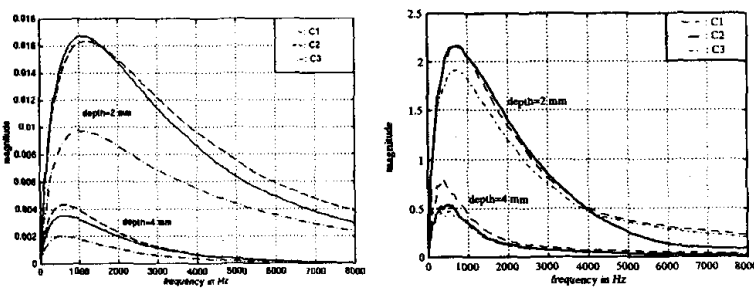

Fig. 6. Magnitude spectra respectively simulation and measurement.
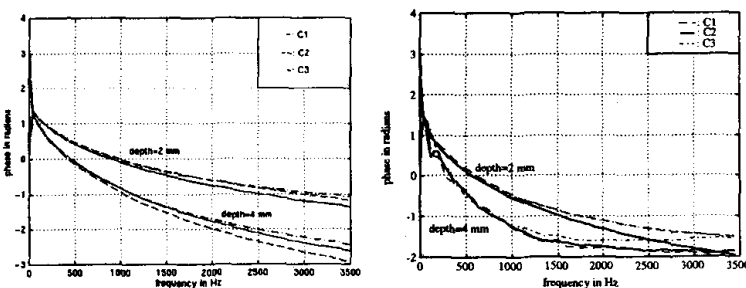

Fig. 7. Phase spectra respectively simulation and measurement. 
$\mathrm{C} 1=2 \mathrm{~mm} \times 2 \mathrm{~mm}, \mathrm{C} 2=4 \mathrm{~mm} \times 1 \mathrm{~mm}, \mathrm{C} 3=1 \mathrm{~mm} \times 4 \mathrm{~mm}$ ), for 2 distinct depths (2 and $4 \mathrm{~mm})$. Both simulations and measurements are made. Applying the CG method, we obtait the impulse responses (Fig.5) for our 6 different cases.

Then magnitude (Fig.6) and phase (Fig.7) spectra can be obtained directly from impulse response using a F.F.T algorithm. There is a good agreement between simulations and measurements. But we can notice on the measurements that, for a given depth the variation due to the shape of the flaw is small.

\section{CHARACTERIZATION OF FLAW}

The purpose of this survey is to characterize different kinds of flaws at several depths. That is to say, three main parameters the depth (material above the flaw), the length, and the height of the flaws have to be found. But looking at the precedent curves it seems difficult to obtain directly the influence of the parameters with the spectra. Hence, we propose to realize a statistic processing of the measurement data. After a first analysis of the results, we select four characterization parameters. The first $f_{m}$ is the frequency which give the maximum magnitude of the magnitude spectrum. The second $a_{m}$ is the value of this magnitude. Then $\varphi$ is the value of the phase at a frequency of $500 \mathrm{~Hz}$. And the last $t_{0}$ is respectively the first passage by zero of the impulse response for the measurement and the time corresponding to the maximum of the impulse response for the simulation. This difference in the choice comes from the difficulty to estimate the time of the maximum on the impulse response obtained by measurement (Fig.5). The choice for $f_{m}, a_{m}$, and $t_{0}$ is due to the fact that they are respectively singular parameters of the magnitude spectra (Fig.6) and of the impulse response (Fig.5).We choose $\varphi$, because of its low variation with the shape of the flaw and strong variation with the depth (Fig.7). Then in the following step, we set up the correlation between the chosen parameters and the characteristics of the flaws (Table I). For this, we realized 32 simulations in order to have a wide range of variation for each characteristic. We used the same depth than before 2 and $4 \mathrm{~mm}$, and the length and the height take the following values $1,2,3$ and $4 \mathrm{~mm}$. Thus, using all the possible combinations for the various lengths and depths, we have 16 different flaws for each depth. For the measurements, we used the 6 cases presented in the above paragraph.

Looking at the correlation matrix, we see a good agreement between simulation and measurement. All the characterization parameters are strongly correlated with the depth except the magnitude $a_{m}$ in the case of simulation. But we have to take into account the influence of the low number

\section{TABLE I: CORRELATION MATRIX}

\begin{tabular}{ccccccc}
\hline & \multicolumn{3}{c}{ simulation } & \multicolumn{3}{c}{ measurement } \\
\hline & depth & height & length & depth & height & length \\
\hline$f_{m}$ & -0.95 & -0.199 & -0.151 & -0.93 & -0.29 & -0.27 \\
\hline$a_{m}$ & 0.754 & 0.264 & 0.481 & 0.98 & 0.1 & 0.05 \\
\hline$\varphi$ & -0.97 & -0.155 & -0.167 & -0.99 & -0.014 & -0.05 \\
\hline$t_{0}$ & 0.975 & 0.028 & 0.187 & 0.99 & 0.022 & 0.01 \\
\hline
\end{tabular}

of studied case on the correlation coefficients for the measurement results. So as a first result, we can say that the flaw depth can be characterized by $t_{0}$ and $\varphi$, and it emerges that among the chosen parameters, none depends only on the length or on the height.

\section{EXTENSION OF THE SURVEY}

In this part, we only work with data obtained with simulation. We realized linear regression on the previous data to set up relations between flaws characteristics and characterization parameters. Then, we compare the estimated value of the parameters with the value of the observations for the 32 different cases (Fig.8). The observations are the real values of the parameters and the number of the observation is one of these 32 different cases. The estimation of $f_{m}$ and $t_{0}$ are not very satisfying and wrong for $a_{m}$.

Hence, on a physical point of view, the phase $\varphi$ presents a linear evolution with the characteristics of the flaws. Using linear regression, only the phase can be used to characterize the flaws. But, we need additional relations to obtain the length $l$, the height $h$, and depth $d$. So we have two possibilities. The first consists in 3 different measurements of the phase $\varphi$ and the second is to use several measurement points. We start with the first possibility. $\varphi$ is the value of the phase for a given frequency. For 3 distinct values of the phase at 3 frequencies $(\mathrm{f}=320 \mathrm{~Hz}, \mathrm{f}=500 \mathrm{~Hz}, \mathrm{f}=800 \mathrm{~Hz}$ ) linear regression gives the following system.

$\left\{\begin{array}{l}\varphi_{320}=1.295-0.0327 l-0.0349 h-0.212 d \\ \varphi_{500}=1.207-0.043 l-0.0399 h-0.280 d \\ \varphi_{800}=1.099-0.0585 l-0.0434 h-0.367 d\end{array}\right.$

Solving this system drives to an estimation of $l, h$, and $d$ for each observation (Fig.9). For $h$ and $d$, results are rather good, but for $l$, we realized a poor estimation. In fact the problem comes from close correlation between phase and characteristics for the 3 different phase values. Therefore, the coefficients in system (1) are not very different, so the values
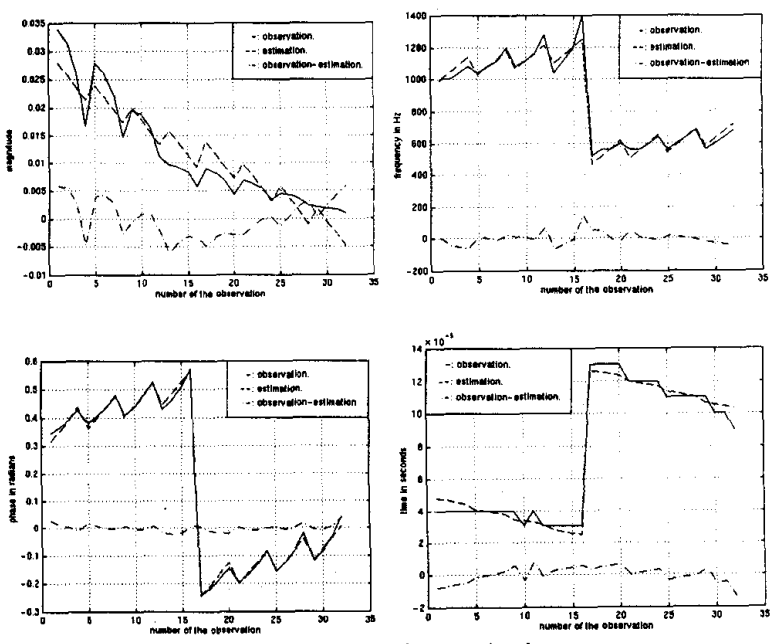

Fig. 8. Estimation of the characterization parameters. 

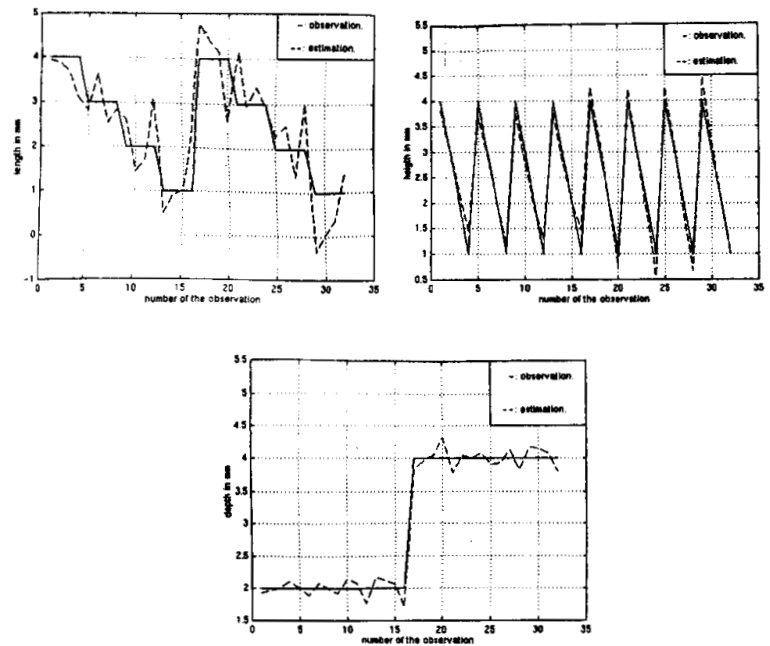

Fig. 9. First estimation of the flaw characteristics.

of the determinant are near zero. In this case, from a low variation of the coefficients may arise important variation of the characteristic $l, h$, and $d$.

For the second possibility, we add an extra measurement point outside the coil near the external winding. With this point, we multiply by two the number of characterization parameters. After an analysis of all possible combinations, we have selected the 3 parameters, which give the best results. These parameters are two distinct values of the phase $\varphi$ $\left(\mathrm{f}=500 \mathrm{~Hz}, \mathrm{f}=800 \mathrm{~Hz}\right.$ ) for the internal point and the time $t_{0}$ for the external point. So, these parameters give a new system:

$\left\{\begin{array}{l}t_{0, \text { external }}=10^{-4} \times(-1.506 l-0.201 h-0.666 d) \\ \varphi_{500, \text { internal }}=1.207-0.043 l-0.0399 h-0.280 d \\ \varphi_{800, \text { internal }}=1.099-0.0585 l-0.0434 h-0.367 d\end{array}\right.$

The new estimations of the parameters (Fig.10) are improved with this set of parameters due to more different coefficients. Furthermore increasing the number of measurement points allowed a wide choice of characterization parameters.

As we are able to estimate the depth of the flaws with satisfying accuracy, we are only going to estimate the length and the height.

So in this last part, we have supposed the depth known and like in the precedent case, we have chosen the best parameters after a study of all the possibilities. These parameters are the phase $\varphi(f=500 \mathrm{~Hz})$ for the 2 measurement points. The precision on the estimation (Fig.11) of height and length is highly increased in this case. So for a known depth, good estimation of the size of the flaw is possible.

\section{CONCLUSION}

This survey presents a deconvolution method in time domain applied to pulsed eddy current NDT. Using finite element method, simulations are realized with 3 different
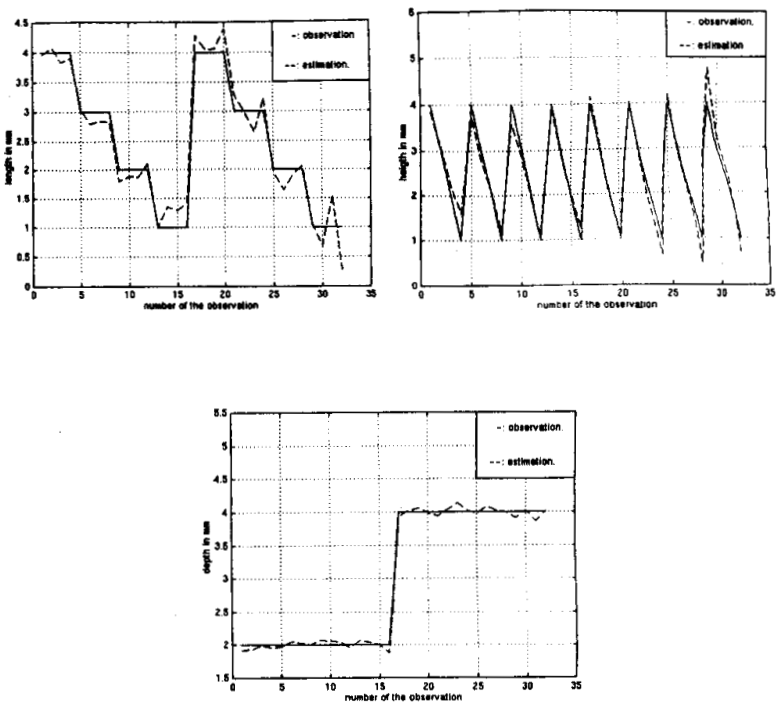

Fig. 10. Second estimation of the flaw characteristics.
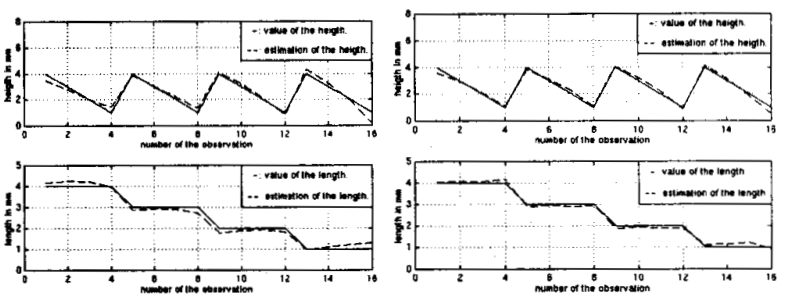

Fig. 11. Length and height estimation for 2 and $4 \mathrm{~mm}$ depth.

flaws at several depths. Such obtained results are employed characterizing the flaws. To confirm these simulation results, a set of measurements has been realized. In the sense of characterization we have shown various estimation results of the flaws characteristics. The perspective for this work is to confirm the modelization results by experimental data.

\section{REFERENCES}

[1] T.K. Sarkar, F.I. Tseng, S.M.Rao, S.A.Dianat, B.Z.Hollmann, "Deconvolution of impulse response from time-limited input and output: theory and experiment," I.E.E.E Transactions on instrumentation and measurement, vol. 34, no 4, pp541-546, December 1985.

[2] F.I. Tseng T.K Sarkar, "Deconvolution of the impulse response of a conducting sphere by the conjugate gradient method," I.E.E.E Transactions on instrumentation and measurement, vol. 35 no 1, pp 105110, December 1987.

[3] F. Thollon," Conception et optimisation de capteurs à courant de Foucault pour la détection de défauts profonds dans des matériaux amagnétiques.," Ph.D.Thesis, Ecole Centrale de Lyon, Janvier 1995.

[4] T.Clauzon, F.Thollon: " De l'utilisation du Gradient Conjugué pour la caractérisation. " PIEMA97, The $3^{\text {rd }}$ intemational Workshop on inverse problems in electromagnetism and acoustic.

[5] T.Clauzon, F.Thollon, " Flaw characterization in Metallic assemblies with pulsed Eddy current NDT", 7ECNDT, Copenhagen DK, 25-29 May 1998. 